\title{
Vícios na estimação da excreção fecal utilizando indicadores internos e óxido crômico em ensaios de digestão com ruminantes
}

\author{
Emanoel Elzo Leal de Barros ${ }^{1}$, Carlos Augusto de Alencar Fontes ${ }^{2}$, Edenio Detmann ${ }^{3}$, \\ Ricardo Augusto Mendonça Vieira ${ }^{2}$, Lara Toledo Henriques ${ }^{4}$, Alberto Magno Fernandes ${ }^{5}$
}

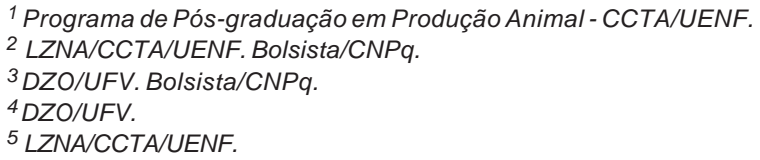

RESUMO - Objetivou-se determinar os vícios de estimação da excreção fecal com base nos indicadores internos matéria seca indigestível (MSi), fibra em detergente neutro indigestível (FDNi), fibra em detergente ácido indigestível (FDAi) e do indicador externo óxido crômico $\left(\mathrm{Cr}_{2} \mathrm{O}_{3}\right)$ em ensaio de digestão com ruminantes. Foram utilizados cinco bovinos mestiços Holandês $\times$ Zebu com peso médio de $240 \mathrm{~kg}$. O experimento constituiu de três períodos experimentais de 12 dias, os seis primeiros destinados à adaptação dos animais. Os animais foram alimentados à vontade com silagem de sorgo e mantidos em baias individuais. Foram adotados seis esquemas de amostragem: 16 coletas diárias, em intervalos de 1 hora e 30 minutos; oito coletas no período diurno a partir das 7 h, em intervalos de 1 hora e 30 minutos; oito coletas no período noturno, a partir das 19 h, em intervalos de 1 hora e 30 minutos; quatro coletas no período diurno, de 3 em 3 horas; quatro coletas no período noturno, de 3 em 3 horas; duas coletas, às 7 h e às 16 h. O óxido crômico foi o indicador com o menor vício de longo prazo, mas superestimou a produção fecal. Os indicadores internos subestimaram esta variável e a FDNi foi o que apresentou menor vício. Para os vícios de curto prazo, todos os indicadores superestimaram a produção fecal: o menor vício de curto prazo foi registrado para a MSi. Dos indicadores testados, a FDNi apresenta o menor vício total, mesmo subestimando a produção fecal.

Palavras-chave: FDAi, FDNi, MSi, óxido crômico, vícios de estimação

\section{Biases in fecal excretion estimation by using internal markers and chromic oxide in digestion trials with ruminants}

\begin{abstract}
The objective of this study was to determine the biases in estimating fecal excretion from the internal markers indigestible dry matter (DMi), indigestible neutral detergent fiber (NDFi) and indigestible acid detergent fiber (ADFi) and the external marker chromic oxide $\left(\mathrm{Cr}_{2} \mathrm{O}_{3}\right)$ in a digestion trial with ruminants. Five crossbred Holstein-Zebu castrated males with $240 \mathrm{~kg}$ average weight were used. The research consisted of three twelve-day experimental periods, from which the first six days were assigned to animal adaptation. The animals were fed sorghum silage ad libitum in individual pens. Six sampling schedules were adopted: 16 daily collections, at 1.5 hourly intervals; eight collections made during daylight starting at 7 a.m. at 1.5 hourly intervals; eight collections made at night starting at 7 p.m. at 1.5 hourley intervals; four collections made in the day every three hours; four collections at night every three hours and two collections, at 7 a.m. and 4 p.m. The chromic oxide was the indicator with the smallest bias in the long term, but underestimated fecal production. The internal markers overestimated this variable and the NDFi presented the smallest bias. For the short term biases, all the indicators over estimated fecal production: the smallest short term bias was registered for MSi. The NDFi was the indicator that presented the smallest total bias, even though it underestimated fecal production.
\end{abstract}

Key Words: indigestible acid detergent fiber, chromic oxide, indigestible dry matter, estimation biases, indigestible neutral detergent fiber

\section{Introdução}

A determinação da digestibilidade tem sido uma das principais preocupações em nutrição animal, pois indica a disponibilidade dos nutrientes no trato gastrintestinal dos animais (Van Soest, 1994). Elevado consumo alimentar e alta excreção fecal em ruminantes implicam trabalhosas coletas e grandes quantidades de amostras produzidas em ensaios de digestão feitos por coleta total, o que leva à utilização de indicadores para estimação da excreção fecal 
e da digestibilidade (Cochran et al., 1986). Os indicadores têm sido utilizados como ferramenta experimental por muitos anos e um amplo número de substâncias tem sido avaliado para estudar a função digestiva dos ruminantes (Merchen, 1993). Indicador é uma substância empregada para monitorar tanto aspectos físicos como químicos da digestão; podem ser de natureza externa (exóticos aos componentes da dieta) ou interna (constituinte intrínseco dos alimentos) e empregado com a finalidade de simplificar os procedimentos de medição nos ensaios de digestão. A determinação da excreção fecal pelo método dos indicadores consiste no emprego de uma substância referência ou índice que possibilite sua recuperação quantitativa nas fezes (Owens \& Hanson, 1992). A estimação da concentração fecal diária de indicadores é obtida por análises de pequenas amostras obtidas manualmente no reto do animal em um ou dois horários fixos do dia. Entretanto, a possibilidade de ocorrerem vícios na estimação desta concentração deve ser considerada.

Entende-se por vício ou viés (bias) o erro sistemático que pode ou não ser detectado na medição de uma variável. O vício na estimação fecal dos indicadores é ocasionado pelas variações nas taxas de produção e expulsão de fezes e pode ser subdividido em dois componentes (Langlands et al., 1963): vício de longo prazo, correspondente à porção do vício de estimação relacionada às falhas na recuperação do indicador empregado; e vício de curto prazo, representado pela porção do vício de estimação fecal relacionada às falhas ou inconsistências no procedimento de amostragem fecal (grab ou spot samples) ou variação ao longo do dia. O conhecimento da magnitude dos vícios é importante para correção das médias experimentais visando à obtenção de uma estimativa mais próxima à obtida com a coleta total de fezes.

Objetivou-se com esta pesquisa quantificar os vícios no processo de estimação da produção diária de matéria seca fecal utilizando-se os indicadores internos matéria seca indigestível (MSi), fibra em detergente neutro indigestível (FDNi) e fibra em detergente ácido indigestível (FDAi) e do indicador externo óxido crômico $\left(\mathrm{Cr}_{2} \mathrm{O}_{3}\right)$.

\section{Material e Métodos}

O experimento foi conduzido nas dependências do Núcleo de Pesquisa em Zootecnia do LZNA/CCTA/UENF utilizando-se cinco bovinos machos não-castrados, mestiços Holandês-Zebu, com peso médio de $240 \mathrm{~kg}$, alojados em baias individuais. A alimentação fornecida foi composta exclusivamente de silagem de sorgo ( $65 \mathrm{~g} \mathrm{~PB} / \mathrm{kg} \mathrm{MS}$; 539 g FDN/kg MS), oferecida à vontade, sempre às 7 h30 admitindo-se sobras de $10 \%$ da quantidade oferecida. Amostras de sobras e do alimento fornecido foram acondicionadas em sacos plásticos e congeladas para posteriores análises. Inicialmente, os animais passaram por um período de 10 dias para adaptação à dieta e ao manejo diário nas baias.

Foram administradas duas doses diárias de óxido crômico de 5 g cada, acondicionados em cartucho de papel, às 8 h e às 17 h, com o auxílio de uma sonda (Hopper et al., 1978). Foi adotado um período adicional de sete dias antes do início do período de coletas fecais para adaptação, com o objetivo de se alcançar o equilíbrio de concentração do indicador nas fezes. O fornecimento de $\mathrm{Cr}_{2} \mathrm{O}_{3}$ foi mantido até o final do período experimental.

As amostras fecais foram coletadas em três períodos sequenciais para amostragem, cada uma com duração de seis dias, com intervalo de cinco dias entre estes períodos de amostragem. Durante esses períodos, foram realizadas coletas totais e pontuais em todos os cinco animais, simultaneamente. As coletas totais foram realizadas no primeiro, terceiro e quinto dias de cada período. As fezes obtidas por coleta total foram pesadas para determinação da produção fecal total diária e homogeneizadas. Foram retiradas posteriormente alíquotas de $10 \%$ do material, que foram acondicionadas em sacos plásticos e congeladas a $-20^{\circ} \mathrm{C}$ para posteriores análises.

Durante o segundo, quarto e sexto dias de cada período de coletas, realizaram-se coletas intermitentes de fezes a partir das 7 h, em intervalos de 1 hora e 30 minutos. Para caracterizar as variações na excreção fecal dos indicadores, foram idealizados seis esquemas de coleta de fezes: 1) amostra composta de 16 coletas diárias, realizadas às $7 \mathrm{~h}$, 8h30, 10 h, 11h30, 13 h, 14h30, 16 h, 17h30, 19 h, 20h30, 22 h, 23h30, 1 h; 2h30, 4 h, 5h30; 2) amostra composta de oito coletas no período diurno, realizadas às $7 \mathrm{~h}, 8 \mathrm{~h} 30,10 \mathrm{~h}$, 11h30, 13 h, 14h30, 16 h, 17h30; 3) amostra composta de quatro coletas no período noturno: às 19 h, 20h30, 22 h, 23h30, $1 \mathrm{~h} ; 2 \mathrm{~h} 30$, 4 h e 5h30; 4) amostra composta de quatro coletas no período diurno, realizadas de 3 em 3 horas: às $7 \mathrm{~h}, 10 \mathrm{~h}, 13 \mathrm{~h}$ e $16 \mathrm{~h} ; 5)$ amostra composta de quatro coletas no período noturno, realizadas de 3 em 3 horas: às $19 \mathrm{~h}$, $22 \mathrm{~h}, 1 \mathrm{~h}$ e $4 \mathrm{~h}$; 6) amostra composta de duas coletas no período diurno, às $7 \mathrm{~h} \mathrm{e} 16 \mathrm{~h}$. As amostras foram acondicionadas e conservadas de forma similar às amostras obtidas por coleta total.

Posteriormente, as amostras de fezes foram descongeladas em temperatura ambiente, secas em estufa de ventilação forçada $\left(60^{\circ} \mathrm{C}\right.$ por 72 horas) (Silva \& Queiroz, 2002), processadas em moinho de facas com peneira de porosidade de $2 \mathrm{~mm}$ e acondicionadas em frascos de polietileno, 
devidamente identificados. O mesmo procedimento foi adotado para as amostras de alimento e sobras e, após o processamento físico, as amostras fecais oriundas das coletas totais e as amostras pontuais ou grab samples obtidas segundo os esquemas de amostragem citados anteriormente foram compostas, proporcionalmente, com base no peso seco ao ar e por animal dentro de cada período de coletas.

As estimativas de excreção fecal foram obtidas pela equação proposta por Smith \& Reid (1955):

$$
\mathrm{EF}=\mathrm{I} /\left[\mathrm{I}_{\mathrm{f}}\right] \text {, }
$$

em que EF (g/dia) corresponde à excreção fecal diária; I (g/dia) representa a dose diária de indicador oferecida/ consumida; $\mathrm{e}\left[\mathrm{I}_{\mathrm{f}}\right]$ equivale à concentração do indicador na matéria seca fecal (g/g).

Após a estimação do teor de matéria seca total (Silva \& Queiroz, 2002), alíquotas de 1,1 g das amostras de alimentos, sobras e fezes (coletas totais e amostrais) foram acondicionadas em duplicata em sacos de náilon de $6 \times 3,5 \mathrm{~cm}$, com porosidade de $50 \mathrm{~mm}$, previamente pesados, devidamente identificados e, em seguida, selados a quente. Os sacos foram então, incubados durante 144 horas no rúmen de um bovino adulto submetido à mesma dieta fornecida aos novilhos durante os períodos de coleta. Após esse tempo, os sacos foram retirados e lavados até a água de lavagem permanecer límpida. Na sequência, foram levados à estufa de ventilação forçada a $60^{\circ} \mathrm{C}$ por 72 horas. Após esse período, foram submetidos à secagem em estufa a $105^{\circ} \mathrm{C}$ por uma hora, retirados, esfriados em dessecador e pesados para determinação da concentração de MSi. Posteriormente, foram submetidos à fervura em detergente neutro (Goering \& Van Soest, 1975), por uma hora, lavados com água quente e acetona, secos e pesados conforme procedimento anterior, a fim de se determinar a FDNi. Após a pesagem, foram novamente submetidos à fervura em detergente ácido (Goering \& Van Soest, 1975), seguindo-se outra vez o procedimento descrito anteriormente para determinação da FDAi. A quantidade dos detergentes utilizada foi de $50 \mathrm{~mL}$ por saco.

Posteriormente, as amostras fecais obtidas por coletas totais e pontuais foram quantificadas quanto ao teor de cromo, com preparação de solução mineral, segundo método descrito por Kimura \& Müller (1957), e a avaliação quantitativa realizada por espectrometria de absorção atômica. Concomitantemente, amostras do óxido crômico utilizado foram avaliadas para quantificar possíveis alterações na pureza do composto.

As avaliações dos vícios de curto (VCP) e longo prazos $(V L P)$ e o vício total $(V T=$ VCP + VLP) foram feitas de acordo com as definições estabelecidas por Langlands et al. (1963). Para isso, foram realizados seis esquemas de amostragem para composição das amostras fecais, de acordo com o disposto a seguir:

$$
\mathrm{VT}=\mathrm{EF}_{\mathrm{A}}-\mathrm{EF}_{\mathrm{R}}
$$

em que VT corresponde ao vício total ( $\mathrm{kg} / \mathrm{dia}), \mathrm{EF}_{\mathrm{A}}$ ( $\mathrm{kg} / \mathrm{dia}$ ) à excreção fecal estimada por intermédio da concentração do indicador em amostras fecais ("grab samples”), e $\mathrm{EF}_{\mathrm{R}}$ à excreção fecal real (kg/dia). Por intermédio de pequena manipulação matemática, tem-se que:

$$
\begin{gathered}
\mathrm{VT}=\left(\mathrm{EF}_{\mathrm{A}-}-\mathrm{EF}_{\mathrm{AR}}\right)+\left(\mathrm{EF}_{\mathrm{AR}}-\mathrm{EF}_{\mathrm{R}-}\right) \\
\mathrm{VT}=\mathrm{VCP}+\mathrm{VLP}
\end{gathered}
$$

em que $\mathrm{EF}_{\mathrm{AR}}$ equivale à excreção fecal estimada pela concentração do indicador em amostra fecal representativa (kg/dia); VCP ao vício de curto prazo ( $\mathrm{kg} / \mathrm{dia})$; VLP ao vício de longo prazo; e $\mathrm{VT}, \mathrm{EF}_{\mathrm{A}}$ e $\mathrm{EF}_{\mathrm{R}}$ conforme definidos anteriormente (Langlands et al., 1963).

Sobre as estimativas de recuperação fecal dos indicadores, construíram-se intervalos de confiança com 95\% de probabilidade utilizando-se a distribuição $t$ de Student (Mood et al., 1974). Neste procedimento empregou-se estimativa da variação residual obtida após ajuste de um modelo estatístico contendo os efeitos de animal e período de coleta fecal.

\section{Resultados e Discussão}

Os valores do vício de longo prazo correspondem às diferenças entre a quantidade de fezes excretada (kg MS fecal/dia), medida pela coleta total, e os valores estimados com base nos indicadores (Tabela 1). O vício de longo prazo representa a diferença entre a excreção fecal real e aquela estimada a partir da concentração do indicador em uma amostra fecal representativa das fezes obtidas por coleta total, e relacionado ao grau de recuperação do indicador.

O óxido crômico superestimou a produção fecal diária em apenas $0,126 \mathrm{~kg} / \mathrm{dia}$, o que representa uma diferença de apenas $5 \%$ entre a produção fecal real e a estimada, o que indica boa recuperação desse indicador, cuja recuperação média foi de 96,92\%. Os indicadores internos subestimaram a produção fecal em $0,419,0,411$ e $0,461 \mathrm{~kg} / \mathrm{dia}$, respectivamente, para a MSi, FDNi e FDAi. Esses vícios corresponderam às respectivas diferenças de 16,63; 16,31 e $18,29 \%$ entre a produção fecal real e a estimada. Os indicadores internos apresentaram recuperações médias de 120,35; 120,80 e 123,19\%, respectivamente, e com limite inferior dos respectivos intervalos de confiança a 95\% superiores a $100 \%$. Esse fato pode decorrer da contaminação do material incubado com material ruminal, conforme ressaltado por Van Soest (1994), ou em decorrência de 
Tabela 1 - Vício de longo prazo (kg MS fecal/dia) para a recuperação fecal estimada com três indicadores

\begin{tabular}{|c|c|c|c|c|}
\hline \multirow[t]{2}{*}{ Indicador } & \multirow[t]{2}{*}{ Média } & \multirow[t]{2}{*}{ Coeficiente de variação $(\%)^{1}$} & \multicolumn{2}{|c|}{$\begin{array}{c}\text { Limites intervalo de confianç } \\
\text { (95\% probabilidade) }\end{array}$} \\
\hline & & & Inferior & Superior \\
\hline Óxido crômico & 0,126 & 122,8 & 0,034 & 0,218 \\
\hline Matéria seca indigestível (MSi) & $-0,419$ & 42,7 & $-0,526$ & $-0,312$ \\
\hline fibra em detergente neutro indigestível (FDNi) & $-0,411$ & 58,6 & $-0,554$ & $-0,268$ \\
\hline fibra em detergente ácido indigestível (FDAi) & $-0,461$ & 51,6 & $-0,602$ & $-0,320$ \\
\hline
\end{tabular}

${ }^{1}$ Variação ajustada para os efeitos de animal e período experimental.

falhas na amostragem das fezes (Langlands et al., 1963). Outra fonte de variação acerca da estimação biológica das frações indigeríveis sob anaerobiose reside na necessidade de incubações de longa duração, por exemplo, com períodos superiores a 70 dias (Van Soest et al., 2005), o que indica que a fração de carboidratos fibrosos potencialmente digestíveis deve ser ao menos dividida em dois componentes com propriedades cinéticas distintas (Ellis et al., 2005; Van Soest et al., 2005). A utilização de tempos menores de incubação, como os de 144 h, assumido como padrão, pode acarretar superestimação do resíduo indigerível e, consequentemente, da produção fecal. Entretanto, o impacto desse fator carece ainda de demonstração experimental.

Com relação aos vícios de curto prazo (Tabela 2), para todas as compostas analisadas, observou-se superestimação da produção fecal diária quando utilizado o óxido crômico. Neste caso, a produção fecal foi superestimada em 0,739 kg/dia ou 29,32\% em média. Para os indicadores internos, os vícios de curto prazo médios foram 0,240 kg/dia para a MSi, 0,112 kg/dia para a FDNi e $0,216 \mathrm{~kg} /$ dia para a FDAi. Esses valores correspondem às superestimativas da produção fecal real de 9,52; 4,44 e $8,57 \%$ para os respectivos indicadores internos. Ressalta-se, porém, que a magnitude dos vícios foi pequena em relação ao total diário de fezes excretado. Os elevados coeficientes de variação (Tabela 1) são reflexo das variações nas concentrações dos indicadores nas amostra pontuais, dentro e entre dias.

O vício de curto prazo representa a porção do vício de estimação fecal relacionada às falhas ou inconsistências no procedimento de amostragem fecal por meio de amostras pontuais (grab ou spot samples) ou flutuações da concentração do indicador nas fezes ao longo do dia (variações dentro do dia). O vício de longo prazo corresponde à porção do vício de estimação relacionada às falhas na recuperação do indicador empregado e pode estar relacionado a problemas na obtenção de amostras representativas das fezes, contaminação das amostras in situ com material ruminal ou erros ligados a procedimentos analíticos. $\mathrm{O}$ vício total equivale ao somatório do VCP e do VLP, ocasionado pelo conjunto de fatores que afetam os vícios de curto e longo prazos.

Apesar de o $\mathrm{Cr}_{2} \mathrm{O}_{3}$ ter apresentado recuperação fecal não diferente de $100 \%$ ( $\mathrm{P}>0,05)$, o vício total médio foi maior para o $\mathrm{Cr}_{2} \mathrm{O}_{3}$ quando utilizados os esquemas de amostragem parciais em substituição à coleta total de fezes (Tabela 3). O valor médio das seis compostas realizadas

Tabela 2 - Médias $(\bar{x})$, coeficientes de variação $\left(\mathrm{CV}^{1}{ }^{1}\right)$ e limites inferiores $(\mathrm{Li})$ e superiores $(\mathrm{Ls})$ dos intervalos de confiança $(\alpha=0,05)$ para os vícios de curto prazo para a recuperação fecal estimada utilizando-se indicadores em diferentes esquemas de amostragem fecal

\begin{tabular}{|c|c|c|c|c|c|c|c|c|c|c|c|c|c|c|c|c|}
\hline \multirow[t]{2}{*}{ Esquema } & \multicolumn{4}{|c|}{$\begin{array}{l}\text { Óxido crômico } \\
\qquad\left(\mathrm{Cr}_{2} \mathrm{O}_{3}\right)\end{array}$} & \multicolumn{4}{|c|}{$\begin{array}{c}\text { Matéria seca } \\
\text { indigestível (MSi) }\end{array}$} & \multicolumn{4}{|c|}{$\begin{array}{c}\text { Fibra em detergente neutro } \\
\text { indigestível (FDNi) }\end{array}$} & \multicolumn{4}{|c|}{$\begin{array}{c}\text { Fibra em detergente } \\
\text { ácido indigestível (FDAi) }\end{array}$} \\
\hline & $\bar{x}$ & $\mathrm{Li}$ & Ls & $\mathrm{CV} \%$ & $\bar{x}$ & $\mathrm{Li}$ & Ls & $\mathrm{CV} \%$ & $\bar{x}$ & $\mathrm{Li}$ & Ls & CV\% & $\bar{x}$ & $\mathrm{Li}$ & Ls & $\mathrm{CV} \%$ \\
\hline 16 coletas diárias & 0,725 & 0,502 & 0,947 & 51,6 & $-0,241$ & $-0,451$ & $-0,032$ & 145,9 & $-0,110$ & $-0,285$ & 0,065 & 266,7 & $-0,221$ & $-0,400$ & $-0,042$ & 136,0 \\
\hline $\begin{array}{l}8 \text { coletas no } \\
\text { período diurno }\end{array}$ & 0,648 & 0,408 & 0,887 & 62,1 & $-0,233$ & $-0,445$ & $-0,021$ & 152,5 & $-0,101$ & $-0,274$ & 0,072 & 287,9 & $-0,202$ & $-0,382$ & $-0,022$ & 150,0 \\
\hline $\begin{array}{l}8 \text { coletas no } \\
\text { período noturno }\end{array}$ & 0,824 & 0,596 & 1,052 & 46,5 & $-0,248$ & $-0,456$ & $-0,039$ & 141,2 & $-0,119$ & $-0,294$ & 0,057 & 248,1 & $-0,236$ & $-0,415$ & $-0,058$ & 126,8 \\
\hline $\begin{array}{l}4 \text { coletas no } \\
\text { período diurno }\end{array}$ & 0,667 & 0,427 & 0,907 & 60,5 & $-0,240$ & $-0,450$ & $-0,031$ & 146,5 & $-0,112$ & $-0,284$ & 0,060 & 258,0 & $-0,208$ & $-0,377$ & $-0,038$ & 136,8 \\
\hline $\begin{array}{l}4 \text { coletas no } \\
\text { período noturno }\end{array}$ & 0,855 & 0,593 & 1,118 & 51,5 & $-0,235$ & $-0,444$ & $-0,026$ & 149,2 & $-0,106$ & $-0,282$ & 0,071 & 280,7 & $-0,222$ & $-0,399$ & $-0,045$ & 134,2 \\
\hline $\begin{array}{l}2 \text { coletas (às } 7 \text { h e } \\
\text { às } 16 \mathrm{~h} \text { ) }\end{array}$ & 0,716 & 0,470 & 0,962 & 57,7 & $-0,243$ & $-0,452$ & $-0,033$ & 145,2 & $-0,122$ & $-0,298$ & 0,055 & 243,4 & $-0,208$ & $-0,378$ & $-0,039$ & 136,5 \\
\hline
\end{tabular}


(0,865 kg/dia) representa uma superestimação em 34,33\% da produção fecal diária, o que resulta em subestimação da digestibilidade. É possível que esse efeito tenha sido acentuado pelas variações de curto prazo, o que incluiria as variações entre dias sucessivos, especialmente considerando que a amostragem pontual (grab samples) foi realizada em apenas três dias por período, ao passo que nas rotinas experimentais são recomendados períodos de coleta de sete dias em média. De acordo com Langlands et al. (1963), alta recuperação está associada a baixo vício de longo prazo e o elevado valor de vício total observado está associado à variação da concentração do indicador entre as amostras pontuais ou grab samples (Tabelas 2 e 3). Para os indicadores internos, houve subestimação da produção fecal, pois foram obtidos valores médios de 0,659 kg/dia para a MSi, 0,522 kg/dia para a FDNi e 0,677 kg/dia para a FDAi. Essa subestimação foi de 26,15; 20,79 e 26,87\% para os respectivos indicadores internos, o que resultou em superestimação da digestibilidade. Esses resultados diferem dos encontrados por Berchielli et al. (2000), que verificaram baixa recuperação da $\mathrm{FDNi}$, subestimando a digestibilidade. Os esquemas de amostragem não influenciaram os vícios estimados, e o aumento do número de amostragens realizadas não implicou redução nos vícios de estimação (Tabela 3).

Hooper et al. (1978) concluíram que duas coletas, aproximadamente às $8 \mathrm{~h}$ e $17 \mathrm{~h}$ foram suficientes para obtenção de estimativas não-viciadas da produção fecal diária por vacas leiteiras em sistema de pastejo. Essas recomendações podem ser confirmadas pelas estimativas obtidas para o perfil nictemeral de excreção do óxido crômico por bovinos em que foram avaliados os mesmos esquemas de amostragem deste trabalho, pois se consi- deradas as concentrações esperadas para o teor desse indicador às $7 \mathrm{~h}$ e às $17 \mathrm{~h}$, a média estimada para o teor de cromo a partir destas duas coletas não diferiu da obtida com a coleta total de fezes (Barros et al., 2007). Isso indica que duas coletas fecais em horários próximos aos mencionados acima permitem predizer com melhor acurácia a produção de matéria seca fecal ao longo do dia (Hooper et al., 1978; Barros et al., 2007).

Com base no esquema dois, a produção fecal foi superestimada em $0,774 \mathrm{~kg} / \mathrm{dia}$ ou $30,71 \%$ quando se utilizou o óxido crômico, ao passo que a MSi, a FDNi e a FDAi levaram à subestimação da produção fecal em 0,652 kg/dia, 0,512 kg/dia e 0,663 kg/dia ou 25,87; 20,32 e $26,31 \%$, respectivamente. Os indicadores internos produziram estimativas viciadas para a produção de matéria seca fecal nos esquemas de amostragem adotados (Barros et al., 2007).

Ao considerar o vício total (Tabela 3), observou-se que o melhor indicador foi a FDNi, com o menor vício de estimação. Esse indicador foi considerado eficiente por Zeoula et al. (2002), que observaram recuperação fecal da FDNi de 101,6\%. Por outro lado, Zeoula et al. (2000) e Ítavo et al. (2002) concluíram que a FDNi subestimou a digestibilidade e apresentou elevados coeficientes de variação. O mesmo foi observado por Freitas et al. (2002), que também não obtiveram bons resultados com o uso da FDNi estimada tanto in vitro como in situ; esses autores observaram que o FDNi in vitro subestimou a produção fecal enquanto FDNi in situ a superestimou. O tamanho de partículas, a natureza da fibra, o período de tempo e o sistema de incubação (in vitro ou in situ) são fontes de variação que devem ser consideradas quando se utiliza FDNi como indicador interno (Zeoula et al., 2002).

Tabela 3 - Médias $(\bar{x})$, coeficientes de variação $\left(\mathrm{CV}^{1}{ }^{1}\right)$ e limites inferiores $(\mathrm{Li})$ e superiores $(\mathrm{Ls})$ dos intervalos de confiança $(\alpha=0,05)$ para o vício total de estimação da excreção de matéria seca fecal (kg MS fecal/dia) com o uso de indicadores em diferentes esquemas de amostragem fecal

\begin{tabular}{|c|c|c|c|c|c|c|c|c|c|c|c|c|c|c|c|c|}
\hline \multirow[b]{2}{*}{ Esquema } & \multicolumn{4}{|c|}{$\begin{array}{l}\text { Óxido crômico } \\
\qquad\left(\mathrm{Cr}_{2} \mathrm{O}_{3}\right)\end{array}$} & \multicolumn{4}{|c|}{$\begin{array}{c}\text { Matéria seca } \\
\text { indigestível (MSi) }\end{array}$} & \multicolumn{4}{|c|}{$\begin{array}{l}\text { Fibra em detergente neutro } \\
\text { indigestível (FDNi) }\end{array}$} & \multicolumn{4}{|c|}{$\begin{array}{c}\text { Fibra em detergente } \\
\text { ácido indigestível (FDAi) }\end{array}$} \\
\hline & $\bar{x}$ & $\mathrm{Li}$ & Ls & CV\% & $\bar{x}$ & $\mathrm{Li}$ & Ls & CV\% & $\bar{x}$ & $\mathrm{Li}$ & Ls & CV\% & $\bar{x}$ & $\mathrm{Li}$ & Ls & CV\% \\
\hline $\begin{array}{l}8 \text { coletas } \\
\text { no período diurno }\end{array}$ & 0,774 & 0,535 & 1,013 & 51,98 & $-0,652$ & $-0,864$ & $-0,440$ & 54,52 & $-0,512$ & $-0,685$ & $-0,339$ & 56,85 & $-0,663$ & $-0,843$ & $-0,483$ & 45,54 \\
\hline $\begin{array}{l}8 \text { coletas no } \\
\text { período noturno }\end{array}$ & 0,950 & 0,722 & 1,178 & 40,34 & $-0,667$ & $-0,875$ & $-0,459$ & 52,49 & $-0,530$ & $-0,705$ & $-0,355$ & 55,58 & $-0,697$ & $-0,876$ & $-0,510$ & 42,98 \\
\hline $\begin{array}{l}4 \text { coletas no } \\
\text { período noturno }\end{array}$ & 0,981 & 0,718 & 1,244 & 44,89 & $-0,654$ & $-0,863$ & $-0,445$ & 53,66 & $-0,517$ & $-0,693$ & $-0,341$ & 57,33 & $-0,683$ & $-0,860$ & $-0,506$ & 43,56 \\
\hline $\begin{array}{l}2 \text { coletas (às } 7 \mathrm{~h} \mathrm{e} \\
\text { às } 16 \mathrm{~h} \text { ) }\end{array}$ & 0,842 & 0,596 & 1,088 & 49,06 & $-0,662$ & $-0,872$ & $-0,452$ & 53,25 & $-0,533$ & $-0,709$ & $-0,357$ & 55,56 & $-0,669$ & $-0,838$ & $-0,500$ & 42,48 \\
\hline
\end{tabular}




\section{Conclusões}

Em comparação aos indicadores internos, o óxido crômico possibilita maior recuperação da matéria seca fecal. Entretanto, sua concentração em amostras pontuais está sujeita a variações que podem resultar em vícios de curto prazo elevados. É necessário, portanto, período de coleta longo o suficiente para reduzir as variações entre amostras e entre dias consecutivos, isto é, superior a três dias, mesmo depois de atingido o equilíbrio de excreção (steady-state) do óxido crômico. Os resultados obtidos com duas coletas diárias são semelhantes aos determinados com maior número de coletas diárias. O óxido crômico apresenta maior vício de curto prazo em comparação aos indicadores internos; por seu turno, os indicadores internos são mais estáveis a curto prazo, mas apresentam elevados vícios de longo prazo. Os indicadores internos subestimam a produção fecal, e a FDNi é o que apresenta menor vício de longo prazo e tempo total, o que proporciona estimação mais exata.

\section{Literatura Citada}

BERCHIELLI, T.T.; ANDRADE, P.; FURLAN, C.L. Avaliação de indicadores internos em ensaios de digestibilidade. Revista Brasileira de Zootecnia, v.29, n.3, p.830-833, 2000.

COCHRAN, R.C.; ADAMS, D.C.; WALLACE, J.D. et al. Predicting digestibility of different diets with internal markers: evaluation of four potential markers. Journal of Animal Science, v.63, n.5, p.1476-1483, 1986.

ELLIS, W.C.; MAHLOOJI, M.; MATIS, J.H. Models for estimating parameters of neutral detergent fiber digestion by ruminal microorganisms. Journal of Animal Science, v.83, p.1591-1601, 2005.

FREITAS, D.; BERCHIELLI, T.T.; SILVEIRA, R.N. et al. Produção fecal e fluxo duodenal de matéria seca e matéria orgânica estimados por meio de indicadores. Revista Brasileira de Zootecnia, v.31, n.3, p.1521-1530, 2002 (supl.)

GOERING, H.K.; VAN SOEST, P.J. Forage fiber analyses. Washington: ARS-USDA Agriculture Handbook, 1975. 379p.
HOPPER, J.T.; HOLLOWAY, J.W.; BUTTS JR., W.T. Animal variation in chromium sesquioxide excretion patterns of grazing cows. Journal of Animal Science, v.46, n.4, p.1098-1102, 1978.

ÍTAVO, L.C.V.; VALADARES FILHO, S.C.; SILVA, F.F. et al. Consumo, degradabilidade ruminal e digestibilidade aparente de fenos de gramíneas do gênero Cynodon e rações concentradas utilizando indicadores internos. Revista Brasileira de Zootecnia, v.31, n.2, p.1024-1032, 2002.

KIMURA, F.T.; MÜLLER, V.L. Improved determination of chromic oxide in cal feed and feces. Journal of Agricultural and Food Chemistry, v.5, n.2, p.216, 1957.

LANGLANDS, J.P.; CORBETT, J.L.; McDONALD, I. et al, Estimation of the faeces output of grazing animals from the concentration of chromium sesquioxide in a sample of faeces. 1. Comparison of estimates from samples taked a fixed times of day with faeces outputs measured directly. British Journal of Nutrition, v.17, n.2, p.211-218, 1963.

MERCHEN, N.R. Digestión, absorción y excreción en los rumiantes. In: CHURCH D. C. (Ed.) El rumiant fisiología digestive y nutrición. 1.ed. Zaragoza: Editora Acribia, 1993. p.191-223.

MOOD, A.M.; GRAYBILL, F.A.; BOES, D.C. Introduction to the Theory of Statistics. 3.ed. Tokyo: McGraw-Hill, 1974. 564p.

OWENS, F.N.; HANSON, C.F. External and internal markers for apprising site and extent of digestion in ruminant. Journal of Dairy Science, v.75, p.2605-2617, 1992.

SILVA, D.J.; QUEIROZ, A.C. Análise de alimentos: métodos químicos e biológicos. 3.ed. Viçosa, MG: Editora UFV, 2002. 235p.

SMITH, A.M.; REID, J.T. Use of chromic oxide as an indicator of fecal output for the purpose of determining the intake of a pasture herbage by grazing cows. Journal of Dairy Science, v.38, n.5, p.515-524, 1955.

VAN SOEST, P.J. Nutrional ecology of the ruminant. 2.ed. Ithaca: Cornell University Press, 1994. 476p.

VAN SOEST, P.J.; VAN AMBURGH, M.E.; ROBERTSON, J.B. et al. Validation of the 2.4 times lignin factor for ultimate extent of NDF digestion, and curve peeling rate of fermentation curves into pools. In: PROCEEDINGS OF THE CORNELL NUTRITION CONFERENCE. 2005, Ithaca. Proceedings... Ithaca, 2005. p.139-149.

WILLIANS, C.H.; DAVID, D.J.; IISMA, O. The determination of chromic oxide in faces samples by atomic absortion spectrophotometry. Journal of Agricultural Science, v.59, n.3, p.381-385, 1962.

ZEOULA, L.M.; KASSIES, M.P.; FREGADOLLI, F.L. et al. Uso de indicadores na determinação da digestibilidade parcial e total em bovinos. Acta Scientiarum. Animal Sciences, v.22, n.3, p.771-777, 2000. 